\title{
Detección y atención precoz de la patología mental en la primera infancia ${ }^{1}$
}

\section{Introducción}

Se sabe que factores orgánicos, neurológicos, ambientales y relacionales pueden influir en el desarrollo emocional del bebé, provocando trastornos psicopatológicos definidos.

Los aspectos preventivos en esta área en la primera infancia han sido escasamente abordados, por la tardanza en la aparición y desarrollo de equipos y profesionales específicos de Salud Mental del Niño y del Adolescente en nuestro país, apareciendo importantes iniciativas al respecto pero que no se han generalizado (Protocolo de salud Mental en Programas del Niño Sano, Protocolo de seguimiento del neonato de alto riesgo, Programa de Detección de R .Social de la C.A.M.); asimismo estos aspectos están englobados, aunque no claramente especificados, en los planteamientos de la Atención Temprana, cuyo gran avance y esfuerzo están plasmados en el Libro Blanco de la Atención Temprana.

En la actualidad, por el contrario, comienza a aparecer un interés creciente y una necesidad de la detección e intervención precoz de trastornos mentales en estas edades, muchos de ellos con características graves como la psicosis, con tendencia a la cronicidad, y que por la precocidad del trastorno abocan a estados deficitarios, relacionados también con patologías psiquiátricas de constatación posterior, como son los trastornos de la personalidad de características psicopáticas o los trastornos borderlaine, cuya clínica en niños y adolescentes puede ser muy abigarrada.

Desde el ámbito de la Salud Mental se da especial relevancia a las alteraciones de las interacciones padresbebé, que sabemos están en el origen de muchos trastornos mentales posteriores, $\mathrm{y}$ que en todo niño con un trastorno mental grave la relación con el entorno está alterada.

El campo del desarrollo emocional y de la psicopatología de la primera infancia es complejo y en su comprensión y abordaje terapéutico requiere una especificidad propia, que debe ser asumida por profesionales y dispositivos específicos en Salud Mental Infanto Juvenil.

Si tenemos en cuenta los intereses y objetivos comunes con otros campos de la salud, con atención temprana, con educación y en el caso, sobre todo de riesgo social, con los servicios sociales, se hace necesaria una coordinación y un funcionamiento interdisciplinar, pero especificando claramente las necesidades de la atención a niños con patología mental, especialmente en el caso de los trastornos generalizados del desarrollo y otras psicopatologías graves de la primera infancia. El trabajo clínico se realiza con intervenciones psicoterapéuticas basadas en las interacciones padres-bebé, antes de los dos años, y sería importante que a

\footnotetext{
${ }^{1}$ Este trabajo es el resultado de las diferentes reuniones tenidas a nivel nacional de especialistas en psiquiatría y psicología infanto juvenil pertenecientes a distintas comunidades autónomas, habiendo sido propiciado este grupo de trabajo por la Sección de Salud Mental Infanto Juvenil de la AEN.
} 
partir de los dos años se pueda también realizar un abordaje grupal. En estos casos se debe dar prioridad al vínculo relacional que el niño pueda establecer y no tanto a una actitud de estimulación. Para ello es preciso contar con un equipo interdisciplinar específico y con unas técnicas también específicas, basadas en el desarrollo emocional y en las interacciones precoces del niño con el entorno.

Son variadas las patologías que pueden provocar trastornos en el desarrollo (parálisis cerebral, síndrome de Down, sordera, ceguera, psicosis...) y, aunque la actuación precoz en muchas de ellas sea similar, la especificidad de los trastornos mentales obliga a la especialización en la formación y en la composición de los profesionales que aborden estas problemáticas. Sin embargo, en la actualidad, aún es muy desigual el desarrollo de las unidades de salud mental infanto juvenil, siendo muy precario en algunas comunidades autónomas, en donde cubren estas deficiencias otros dispositivos fundamentalmente desde el ámbito de educación y de servicios sociales. En el caso que nos ocupa, los equipos de atención temprana son los que en muchos casos atienden en estas edades la psicopatología grave, que lleva aparejada por lo general retrasos psicomotores. Es necesario, pues, potenciar y desarrollar los equipos de salud mental infanto juvenil en las distintas comunidades autónomas, y en aquellos casos en los que los equipos de atención temprana sean los que están bien implantados asumiendo este campo psicopatológico, dotarlos con profesionales especialistas en psiquiatría y psicología clínica del niño y del adolescente. En ambos casos se hace necesario una formación especifica en el desarrollo emocional y en la psicopatología de la primera infancia, además de ser imprescindible la creación de hospitales de día y/o unidades de tratamiento para el abordaje terapéutico.

Es evidente que en todo niño que nace con una discapacidad o un riesgo elevado de padecerla, de la causa que sea, está amenazado en gran medida su mundo relacional, por lo que cualquier dispositivo que trabaje con estos niños no puede olvidar esta dimensión, especialmente en los trastornos mentales severos, siendo uno de los pilares donde se asienta la enfermedad, estando además muy implicado el desarrollo emocional y no sólo el cognitivo, existiendo una relación con el entorno muy alterada.

Es desde esta perspectiva desde la que planteamos protocolos de salud mental en la primera infancia, haciendo mayor hincapié en la detección y abordaje precoz de la patología mental grave.

\section{Protocolo para la detección de trastornos mentales en la primera infancia ${ }^{2}$}

Debido a la importancia que para el desarrollo psíquico del niño tienen unas bases emocionales sólidas, unidas a la diversidad de criterios para la evaluación y orientación terapéutica de los problemas psíquicos (dependiendo estas del origen y la formación del profesional que primeramente atienda al niño, bien sea pediatra, neurólogo, psicólogo, psiquiatra, profesional de atención temprana o de educación), se hace necesario la elaboración de una guía clara, que permita la unificación de criterios entre diferentes profesionales, favoreciendo la detección precoz de trastornos mentales en la primera infancia y su derivación a los dispositivos específicos de salud mental infanto juvenil.

\footnotetext{
${ }^{2}$ Basado en el Protocolo para la detección del Riesgo Social en el Recién Nacido, utilizado en el Servicio de Neonatología del Hospital Clínico de Madrid y en el Protocol de Salut Mental Infantil i Juvenil del Programa de Seguiment del Nen Sa, editado por el Servei Catalá de la Salut.
} 
INFORMES

Fundamentalmente la detección e intervención precoz en niños con un trastorno mental severo se hace imprescindible por lo que conllevan estas enfermedades de alteración del desarrollo emocional y cognitivo, y que abocan por lo general a deficiencias múltiples de instauración precoz.

Sin embargo, estos trastornos son detectados tardíamente, pues se "pierde" mucho tiempo de tratamiento mientras se realiza el proceso diagnóstico, que suele ser un peregrinar del niño y su familia por diferentes especialistas. Además, en muchos casos, son considerados como problemas deficitarios o de diferentes discapacidades, por lo que no reciben un tratamiento específico.

En relación a los factores psicopatológicos, algunos de ellos ya pueden estar incidiendo desde el embarazo y pueden ser detectados, por lo que es importante tenerlos en cuenta en los programas de preparación al parto y consultas prenatales.

En los servicios de Neonatología, donde se atiende a los niños con alto riesgo orgánico, es posible observar los problemas de las interacciones padres-bebé que suelen crearse, debido fundamentalmente a la situación en la que se encuentra el niño, y empezar a trabajar sobre ello.

Las primeras manifestaciones de estos trastornos psicopatológicos en los niños muy pequeños suelen ser fundamentalmente de naturaleza somática y/o psicosomática, en un principio, por lo que se recurre primeramente a los servicios de Pediatría y/o de Neuropediatría, que se encuentran en una posición privilegiada para la detección precoz.

Por otra parte, en los centros de salud de atención primaria se desarrollan los programas de seguimiento a los niños sanos, siendo un lugar prioritario para la detección. Igual importancia tienen los servicios sociales de base que, por lo general, desarrollan programas para la infancia, especialmente cuando el niño pueda estar en riesgo o desamparo. Uno de los factores importantes de riesgo es la psicopatología de los padres, por lo que desde los equipos de salud mental de adultos se debe desarrollar un trabajo en este sentido. Por ultimo, las escuelas infantiles son lugares donde suelen detectarse muchas alteraciones relacionales y del desarrollo psicomotor de los niños, así como en los equipos de atención temprana que, por su propio trabajo, atienden niños con diferentes problemas en su desarrollo y que por tanto pueden presentar trastornos psicopatológicos.

Para que este protocolo mejore su eficacia se propone que se complemente con:

- Los protocolos ya existentes: "detección de alto riesgo psicosocial en el neonato", "detección precoz del niño sordo y del niño ciego", los "programas de preparación al parto" y con el "programa del niño sano".

- La creación, en cada área sanitaria, de circuitos claros que agilicen las exploraciones que se realicen (neuropediátricas, psicológicas, psíquicas y sociales) para poder llegar a un diagnóstico lo más claro y rápido posible. De este modo se garantizaría que todo niño detectado con una posible patología mental severa o semiología de alarma 
tenga este estudio diagnóstico realizado, pero habría que asegurar que el mismo se haga sin que interfiera en la intervención precoz específica, y siempre en el seno de una actuación coordinada de los diferentes especialistas.

- La formación de "comisiones técnicas" interprofesionales en cada zona sanitaria, que lleven a cabo reuniones periódicas donde se debata sobre cada caso, unificando y coordinando actuaciones, tanto psicoterapéuticas, como sociales, educativas y otras.

- La coordinación de la actuación de los demás especialistas por parte del profesional responsable que, en los casos de una patología o una disfunción en Salud Mental, ha de ser un especialista del equipo de S.M.I.J.

- La formación continuada para los distintos profesionales que atienden al niño en esta primera etapa.

- La realización de un registro específico de estos niños, que pueda valer para estudios epidemiológicos futuros.

\section{Criterios de detección}

Factores de riesgo psicopatológico

Se consideran factores de riesgo todas aquellas circunstancias del niño o de su entorno que puedan conllevar una mayor posibilidad de trastorno psicopatológico que la que se observa en la población general.

Un factor de riesgo, por sí solo, no tiene por qué ser significativo, pero la asociación de varios potencian ese riesgo, por lo que es importante su detección para una intervención especializada lo antes posible, aunque nunca hay que olvidar que no todos los niños tienen la misma vulnerabilidad (basada en mecanismos biológicos y psicológicos).

Asimismo son muy importantes los factores protectores: es decir, factores personales o ambientales que pueden amortiguar el riesgo.

Personales: el más importante, la resistencia o capacidad de adaptarse a situaciones adversas; esta relacionada con factores genéticos, como el temperamento y la apariencia física, especialmente en las primeras edades; también es importante la inteligencia a medida que se va creciendo. Percepción de autoestima y autoeficacia de uno mismo son los dos factores que más se utilizan, para detectar factores de protección personales.

Ambientales: se refiere a recursos sociales de apoyo internos o externos a la familia, pueden ser desde la escuela a un familiar próximo o adulto cercano (cuidador) al niño que le proporciona una base de seguridad afectiva.

En niños mayores son importantes los amigos; tener amigos en los que apoyarse puede reducir el riesgo, o, dependiendo del tipo de amistades, aumentarlo.

1. Circunstancias del embarazo:

- Embarazo en preadolescentes y adolescentes, con dificultades de relación y si se ignora la identidad del padre (sospecha de abuso sexual). 
INFORMES

- Hijo no aceptado al final del embarazo.

- Embarazo de riesgo (no controlado/ oculto, hospitalización o inmovilidad prolongada, hiperemesis gravídica, consumo de alcohol, drogas...)

- Embarazos no suficientemente espaciados y/o familias con número elevado de hijos.

- Embarazo por técnicas de reproducción asistida.

2. Circunstancias perinatales:

- Prematuros - Postmaduros

- Sufrimiento fetal

- Nacido con defectos o malformaciones

- Hospitalización prolongada o repetidas en el primer año

- Separación prolongada madre-hijo en este período

3. Características temperamentales como factor de riesgo

Temperamento difícil (ya observable en recién nacidos)

- Niños complicados/problemáticos en las primeras semanas. Es un factor de riesgo moderado, inespecífico que aumenta cuando el niño "difícil" vive en un ambiente familiar patológico, conflictivo o incoherente y poco estimulante desde el punto de vista educativo.

- Humor negativo (irritable, colérico, triste..)

- Arrítmico (vigilia/sueño, alimentación, eliminación)

- Difícil de controlar y apaciguar

- Reacciones intensas, desmesuradas.

- Adaptación muy lenta a situaciones nuevas (personas objetos, alimentos, etc..)

- Tendencia al retraimiento o inhibición social

4. Factores familiares

- Padre/madre con problemas de adicción (drogadicción/alcoholismo, ludopatía).

- Familias con historia de violencia (física o verbal) y/o maltrato infantil (entre adultos y adultos-niños/as).

- Padres muy jóvenes (menores de edad) o mayores.

- Situación de ruptura familiar o familias monoparentales.

- Padre/madre con enfermedad orgánica crónica.

- Padre / madre con minusvalía

- Padre/madre con historia de enfermedad mental.

- Madre con depresión posparto intensa y/o prolongada.

- Madre con psicosis puerperal.

- Pérdida significativa de un familiar.

- Niño en acogimiento familiar y/o en adopción. 
5. Relación padres- hijo/ a:

- Separación de la madre en el período neonatal precoz, con imposibilidad absoluta de contacto físico entre ambos.

- Desinterés o rechazo por el recién nacido (estar con él, alimentarle, vestirle..) y/o escasas visitas cuando el niño/a está ingresado que, además, pueden ser inconsistentes y cortas.

- Maltrato físico y/o sexual al niño.

- Padres con actitudes incoherentes, indiferentes o con excesiva ansiedad ante las responsabilidades de crianza de los hijos / as.

- Padre/madre con comportamientos llamativos (1lanto, gran tristeza, excitación, continuas preguntas, incomunicación total, contradicciones, ocultamientos, negativa a hablar con otros profesionales...).

- Discontinuidad en los cuidados parentales (cambios frecuentes de personas, de cuidadores).

- Ausencia del padre en el cuidado del bebé.

6. Factores socio- económicos:

- Familias con problemas económicos (precariedad económica, carencia de domicilio...)

- Aislamiento social (emigración, falta de apoyos familiares y sociales...)

- Otros hijos con medidas de protección (guarda o tutela).

- Dificultad social (prostitución, delincuencia, narcotráfico, mendicidad, privación de libertad...)

\section{Signos de alarma}

Se consideran signos de alarma cualquier dato de la observación clínica que puede ser una manifestación de una alteración que podría hacerse evidente en aquel momento o en una etapa ulterior de la evolución del individuo.

(Los signos marcados con * pueden ser indicadores de trastornos psicopatológicos graves (autismo, otras psicosis infantiles o trastorno generalizado del desarrollo y disarmonías evolutivas severas)

\section{Trastornos de la alimentación y/o deglución}

Se excluyen los trastornos de la conducta alimentaria provocados por actitudes inadecuadas de la familia.

- Inapetencia (ingerir muy poca cantidad de alimento o duración de mamada más de 60 minutos)

- Rechazo de alimentos sólidos (después de los 6-8 meses) y dificultad para masticar.

- Vómitos de origen funcional: 
INFORMES

- Cuando se acompañen de reacciones emocionales intensas (por ejemplo de angustia) o de comportamientos, generalmente de oposición, que el niño practica para vomitar (por ejemplo introducirse el dedo o algún otro objeto en la boca)

- Después de comer, en niños inapetentes que es necesario alimentar pasivamente, caracterizados por la salida lenta de lo que han ingerido.

- Mericismo* y pica:

- Mericismo *: rumiación más vómito (alimento retornado a la boca). Acostumbra a aparecer en el segundo semestre de vida.

- Pica: Ingestión de sustancias no alimentarias (por ejemplo tierra, papeles, tizas, etc..)

- Trastornos digestivos de origen funcional:

- Estreñimiento (regularmente)

- Diarreas (regularmente)

- Niño que, habitualmente, es forzado a alimentarse.

2. Trastornos del sueño

- Dificultades para dormir, a partir de los primeros meses, o al menos durante 4 semanas, casi todas las noches.

- Insomnio:

- Expresado en forma de llantos (durante 4 semanas casi todas las noches)

- Acompañado de actividades motoras elaboradas (balanceo, cabezazos)

- Gran insomnio* (a veces acompañado de rechazo sistemático del contacto, desvinculación, apatía)

- Silencioso * (niños que pasan horas de noche o de día despiertos, sin manifestar nada, y con los ojos abiertos).

- Hipersomnia (somnolencia prolongada y poca reactividad e indiferencia).

\section{Trastornos del comportamiento}

(Cuando se presentan regularmente, cuando siguen con intensidad fuerte o mediana y cuando desbordan las capacidades de tolerancia y contención de los padres o de los educadores).

- Inestabilidad

- Conducta hiperactiva.

- Oposicionismo-negativismo sistemático

- Conductas agresivas atípicas *:

- Autolesion, golpes en la cabeza, agresión peligrosa a sí mismo o a otros.

- Deambulación sin meta*.

- Intolerancia a los cambios *, con reacción intensa de angustia.

- Baja tolerancia a la frustración (niño difícil de calmar)

- Conductas autoestimulatorias* (balanceos, movimientos reiterativos de objetos...)

- Movimientos estereotipados*. 
- Sin sentido del riesgo (accidentes repetidos)

- Malestar desproporcionado ante pequeños cambios del entorno.

- Preocupación excesiva por detalles o formas de diversos objetos *.

- Tendencia a la inhibición conductual (después del primer año de edad):

- En situaciones nuevas, especialmente delante de personas que no le resultan familiares (delante del medico, etc).

- Tarda exageradamente en responder

- Se asusta excesivamente de los desconocidos

- Se aferra y se coge continuadamente y excesivamente a la madre o la figura sustituta.

- Todo esto se acompaña de activación vegetativa.

- Trastorno del control de los impulsos (masturbación compulsiva, cleptomanía, crisis de cólera...)

4. Miedos y disfunciones de las relaciones sociales

- Miedos con evitación sistemática de situaciones: noche, oscuridad, algunos animales, personas desconocidas, situaciones nuevas... (Cuando se presenta regularmente y con intensidad mediana o fuerte a partir de los 2-3 años de edad)

- Fobias graves y raras*.

- Hipersensibilidad a ruidos cotidianos.

- Timidez intensa con rechazo del contacto.

- Problemas importantes con los iguales (agresividad excesiva y/o aislamiento).

- Ausencia de contacto visual *:

- No mira, evita sistemáticamente la mirada de personas, no mira objetos, no hace seguimiento visual, mirada perdida.

- Ausencia de sonrisa o sonrisa infrecuente *:

- Niño absorto en sí mismo, con un estado de ánimo que se caracteriza por irritabilidad y depresión, más que por retraimiento.

- Falta de respuesta a estímulos sociales *:

- Afectos inapropiados al contexto de las situaciones, inclinación exagerada a las personas o a la inversa, ausencia de contactos interpersonales, insociabilidad.

\section{Anomalías en el juego}

- Inhibición:

- Niño que no juega.

- No inicia el juego simbólico (a partir de los 2- 3 años).

- No utiliza los juguetes para representar escenas, se contenta con ordenarlos

y manipularlos sin establecer relación con su significado *.

- Juego pobre y/ o estereotipado *.

- Juego muy sangriento y cruel. 
INFORMES

7. Problemas en el desarrollo del lenguaje, psicomotor y cognitivo

- Retraso en la adquisición del lenguaje comprensivo * y expresivo.

- Ausencia de deseo comunicativo*.

- Dificultad en el uso pronominal de yo * (a partir de dos años).

- Habla siguiendo un patrón peculiar *: Ecolalia, inversión de pronombres, hiperinterrogación, verborrea.

- No desarrollo de la atención compartida.*

- No desarrollo, inicio y/o consolidación de la permanencia de objeto *.

- Dificultad en la simbolización (reconocimiento de imágenes, fotos...) *.

- Retraso psicomotor generalizado.

- Hipotonía en los primeros meses o, en ocasiones hipertonía, y dificultad del ajuste postural. *

- Pérdida brusca de las funciones motoras adquiridas *.

- Poca expresividad facial (cara triste, indiferente) y/o rigidez facial .*

- No echa de menos a los padres, se queda sólo o con extraños a partir de los 10-12 meses.

- Retraso (o ausencia) en la sedestación, volteos, rastreo y en la bipedestación.

- Retraso en el control de esfínteres.

- Precocidad excesiva en el control de esfínteres.

- Alteraciones de la atención (fija, dispersa, lábil).

- Retrasos en el aprendizaje escolar.

8. Trastornos de la interación padres-bebé ${ }^{3}$

\section{Relación sobreinvolucrada}

Excesivo involucramiento físico y/o psicológico.

- El progenitor obstaculiza las metas y deseos del infante.

- El progenitor controla excesivamente.

- El progenitor formula exigencias inadecuadas para el nivel de desarrollo del niño

- El infante parece difuso, no enfocado e indiferenciado.

- El infante despliega conductas sumisas, excesivamente obedientes o, a la inversa, desafiantes.

- El infante puede carecer de habilidades motrices y/o expresividad lingüística.

\section{Relación subinvolucrada}

La relación subinvolucrada se caracteriza por un involucramiento auténtico o conexión con el infante sólo esporádico o infrecuente.

- El progenitor insensible y/o no responde a las señales del infante.

\footnotetext{
${ }^{3}$ Para facilitar la unificación de criterios se utiliza una adaptación de la clasificación de los trastornos de la relación de la "Clasificación Diagnostica (de 0-3) de la salud mental y desórdenes en el desarrollo de la infancia y niñez temprana”. Ed. Paidós
} 
- Falta de consistencia entre las actitudes expresadas acerca del infante y la cualidad de las interacciones reales.

- El progenitor ignora, rechaza o no conforta.

- El progenitor no refleja los estados emocionales internos del infante.

- El progenitor no protege adecuadamente.

- Se observan interacciones insuficientemente reguladas o mal interpretadas por el progenitor.

- Progenitor e infante parecen estar desligados.

- Aparentemente el infante no es cuidado física y/o psicológicamente.

- El infante puede parecer retrasado o precoz en las aptitudes motrices y lingüísticas.

\section{Relación ansiosa / tensa}

Esta relación se caracteriza por interacciones tensas, restringidas, con poco disfrute distendido o mutualidad.

- El progenitor puede ser sobreprotector e hipersensible.

- La manipulación del niño puede ser torpe o tensa.

- En las relaciones aparecen algunas interacciones verbal o emocionalmente negativas

- Es pobre la adecuación entre los temperamentos.

- El infante puede ser muy obediente o ansioso.

\section{Relación colérica / hostil}

Esta relación se caracteriza por una interacción ruda y abrupta, a menudo carente de reciprocidad emocional.

- El progenitor puede ser insensible a las señales del infante.

- La manipulación del niño es abrupta.

- El infante puede parecer asustado, ansioso, inhibido, impulsivo o difusamente agresivo.

- El niño puede presentar conducta desafiante o resistente con el progenitor.

- Conductas exigentes o agresivas con el progenitor.

- El niño puede presentar conductas temerosas, vigilantes y evitativas.

- El niño puede presentar una tendencia a la conductas concretas más que al desarrollo de la fantasía e imaginación.

\section{Relación mixta.}

Combinación de los rasgos descritos en los apartados anteriores.

\section{Criterios de actuación}

Ante la observación de factores de riesgo y/ o de señales de alarma es importante:

- Hacer un seguimiento mayor del niño y su familia. 
- Si hay varios factores de riesgo y/ o señales de alarma, llevar el caso a interconsulta con el equipo de salud mental infanto- juvenil, o su derivación al mismo.

- Si aparecen factores de riesgo marcados con *, la derivación ha de hacerse urgente.

\section{Exploración diagnóstica}

Esta exploración sería necesaria para los niños con sospecha de patología mental grave (espectro autista y otras psicosis infantiles o trastornos generalizados del desarrollo, disarmonías evolutivas severas)

Es importante, para este tipo de exploración, crear espacios de coordinación entre los distintos Servicios para establecer protocolos de actuación, que aseguren una valoración diagnóstica múltiple y clara, que evite trasmitir distintas concepciones a la familia.

Dependiendo de la sospecha de la patología inicial, el clínico valorará qué tipo de pruebas de las enumeradas han de realizarse:

Exploración neuropediátrica (por especialista especifico)

- Analítica sanguínea:

Screening de aminoácidos. Ácido úrico. Calcio. Fósforo. Magnesio. Ácido láctico. Ácido pirúvico y C.P.K. Título de anticuerpos a herpes-virus. Cariotipo (ver la necesidad de estudios de genética molecular). Urea. Amonio. Pruebas Tiroideas. Valorar títulos V.I.H.

- Analítica urinaria:

Ácido úrico. Magnesio. Calcio. Fósforo. Creatinina. (Screening aminoácidos en orina de $24 \mathrm{~h}$.)

- Estudios complementarios:

Examen neurológico básico

EEG. Valorar la necesidad de realizar: TAC cerebral. Resonancia magnética cerebral.

Evaluación ocular completa: Incluir potenciales evocados visuales.

Evaluación otológica completa: Incluir potenciales evocados auditivos.

Estudio de posibles intolerancias a alimentos.

Búsqueda de antecedentes pre y perinatales (premadurez, postmadurez, hemorragia cerebral...)

Exploración psíquica (Por especialista especifico de SMIJ)

- Valoración del desarrollo psicomotor.

- Observación de signos de alarma: relación con los padres, con el entorno, capacidad de simbolización, manejo de la agresividad y del afecto, capacidad de frustración, signos de ansiedad y/o depresivos, signos de desestructuración psicótica...

- Valoración de las capacidades cognitivas.

- Evaluación del lenguaje.

- Evaluación de la dinámica familiar. 
PARTICIPANTES:

- Carmen González Noguera

- Marta Peral

- J. Teresa Armas Falcón

- Elena Cortés Rabal

- Ignacio Avellanosa Cano

- Roque Prego Dorca

- Margarita Vidal Lucena

- Paloma San Román Villalón

- Mercedes Valle Trapero

- Lucía Alvárez-Buylla Bustillo

- Ma Teresa de Miguel Martínez

- Esther Guerrero Buitrago

- Carmen Manjón Ortega

- Xavier Tapia Lizeaga

- Dolores Izuzquiza

- Margarita Ibáñez Fanés

- Alfonso Verdú

- Ana Ma Jiménez Pascual
-Hospital Insular de Gran Canaria.

-Hospital del Niño Jesús- Madrid

-U.S.M. LASSO (Las Palmas de G.Canaria)

-U.S.M CANALEJAS (Palmas de G. Canaria)

-Hospital Clínico (Madrid)

-Serv. Salud Mental Infantil (Santander)

-Hosp.S.Juan de Dios (Barcelona)

-Hosp. Perpetuo Socorro (Albacete)

-Hospital Clínico (Madrid)

-C.S.M. Ciudad Lineal (Madrid)

-Hosp. Santa Cristina (Madrid)

-Hospital Santa Cristina (Madrid)

-U.S.M.I.J. (Alava)

-ALTXA (Bilbao)

-Madrid

-Hosp. S. Juan de Dios (Barcelona)

-Hosp. Virgen de la Salud (Toledo)

-U.S.M.I.J Alcázar de S. Juan (C.Real)

\section{BIBLIOGRAFÍA}

- "Clasificación Diagnostica (de 0- 3) de la salud mental y desórdenes en el desarrollo de la infancia y niñez temprana". Ed. Paidos.

- Protocolo para la detección del Riesgo Social en el Recién Nacido, Servicio de Neonatología del Hospital Clínico de Madrid

- "Protocol de Salut Mental Infantil i Juvenil" del Programa de Seguiment del Nen Sa, editado por el Servei Catalá de la Salut.

- Grupo de Atención Temprana. Libro Blanco de Atención Temprana Autor:

Real Patronato de Prevención y de Atención a Personas con Minusvalía.

* Coordinadora del grupo de trabajo.

e-mail: usmas@local.jccm.es 